\title{
Clinicopathological and genetic characteristics associated with brain metastases from lung adenocarcinoma and utility as prognostic factors
}

\author{
HIROMASA KOBAYASHI ${ }^{1,2}$, MAKOTO HAMASAKI ${ }^{1}$, TAKASHI MORISHITA ${ }^{2}$, MASAYO YOSHIMURA ${ }^{1}$, \\ MASANI NONAKA ${ }^{2}$, HIROSHI ABE ${ }^{2}$, TOORU INOUE ${ }^{2}$ and KAZUKI NABESHIMA ${ }^{1}$ \\ Departments of ${ }^{1}$ Pathology and ${ }^{2}$ Neurosurgery, Faculty of Medicine, Fukuoka University, Fukuoka 814-0180, Japan
}

Received September 2, 2017; Accepted May 24, 2018

DOI: $10.3892 / \mathrm{ol} .2018 .9225$

\begin{abstract}
Brain metastases (BM) are common in patients with lung adenocarcinoma, and represent a significant cause of morbidity in the disease. A more comprehensive understanding of the clinicopathological characteristics that serve as prognostic factors for survival in patients with BM from lung adenocarcinoma may aid in informing treatment strategies for this patient population. In the present study, clinicopathological factors, including EGFR mutation status, were evaluated in 59 patients who were diagnosed with BM from lung adenocarcinoma, and underwent BM resection between January 1985 and December 2014 at Fukuoka University Hospital. The most frequent subtype of BM from lung adenocarcinoma was solid adenocarcinoma $(57.6 \%)$, followed by papillary adenocarcinoma $(22.0 \%)$ and acinar adenocarcinoma (18.6\%). A total of 14 patients $(23.7 \%)$ exhibited EGFR mutations, which were significantly associated with female sex $(9 / 14,64.3 \%)$, non-smoker status $(8 / 14,57.1 \%), \mathrm{BM}$ in the frontal lobes $(9 / 14$, $64.3 \%)$ and papillary adenocarcinoma $(5 / 14,35.7 \%)$. Statistical analysis revealed a significant association between non-smoker status and BM in the frontal lobes, and more favorable disease prognosis. The results of the present study suggest that
\end{abstract}

Correspondence to: Dr Makoto Hamasaki, Department of Pathology, Faculty of Medicine, Fukuoka University, 7-45-1 Nanakuma, Fukuoka 814-0180, Japan

E-mail: makotoha@fukuoka-u.ac.jp

Abbreviations: ALK, anaplastic lymphoma kinase; BM, brain metastases; ECM, extracranial metastasis; EGFR, epidermal growth factor receptor; GPA, graded prognostic assessment; H\&E, hematoxylin and eosin; KPS, Karnofsky performance score; NSCLC, non-small cell lung cancer; OS, overall survival; RPA, recursive partitioning analysis; TKI, tyrosine kinase inhibitor; TTF-1, Thyroid transcription factor-1; WBRT, whole brain radiation therapy; WHO, World health organization

Key words: brain metastases, lung adenocarcinoma, prognostic factor, epidermal growth factor receptor mutation, histopathological subtype histological and genetic analysis of tissue from BM provides information useful for managing treatment of patients with resectable BM arising from lung adenocarcinoma.

\section{Introduction}

The frequency of brain metastases (BM) from non-small cell lung cancer (NSCLC) exceeds $20 \%$ at the time of diagnosis of the primary tumor, and a significant proportion of patients develop BM over the course of disease $(1,2)$. In recent years, the incidence of BM in patients with NSCLC has increased, due to improved survival rates resulting from the molecular-targeting therapy and improvements in diagnostic neuroimaging modalities, including magnetic resonance imaging and positron emission tomography (3). Adenocarcinoma is the most common subtype of NSCLC, occurring in $~ 40 \%$ of all patients, and is characterized by rapid progression, early distant metastasis and a significantly higher incidence of BM compared with other subtypes of NSCLC (4-6). BM are considered to be a poor prognostic factor in NSCLC, and are associated with median overall survival (OS) times of 4-7 weeks in untreated patients (6-8). In patients with BM, recursive partitioning analysis (RPA) and the graded prognostic assessment (GPA) score are still the most widely accepted models for evaluation of disease prognosis $(6,9)$.

In lung adenocarcinoma, mutations in the epidermal growth factor receptor $(E G F R)$ gene have been identified as a driver mutation promoting tumor growth (10-12). Therapies targeting mutant EGFR protein are associated with relatively high overall response rates, and have been established as standard-of-care treatments (10-12). Furthermore, EGFR-targeted therapies have demonstrated efficacy in the treatment of BM from NSCLC (13-15).

Histological and genetic evaluation is essential in identifying patients with NSCLC that may benefit from EGFR-targeted therapies. In cases in which tissue sampling from the primary tumor is difficult, tissue samples from BM lesions may provide a useful alternative for evaluation. In the majority of previous reports, histological analysis and genetic evaluation in patients with NSCLC have been performed using tissue from the primary tumor only (10-15). There are a few reports describing a histopathological and genetic evaluation 
using tissue samples from BM and the primary lung adenocarcinoma together $(16,17)$. Given evidence that the presence of EGFR mutations is a positive prognostic factor in patients with BM from lung adenocarcinoma (13-15), it is feasible that analysis of tissue from BM may be used to guide more aggressive and effective treatments for patients with $E G F R$ mutation-positive NSCLC.

In the present study, the clinical features and histopathological subtypes of BM from lung adenocarcinoma in a patient population from a single institution were retrospectively evaluated in order to identify prognostic factors. In addition, an evaluation of EGFR mutations and their association with disease prognosis was assessed. The results of the present study suggest that the analysis of tissue from BM may inform the treatment of patients with metastatic brain lesions arising from lung adenocarcinoma.

\section{Materials and methods}

Study design and patients. A total of $232 \mathrm{BM}$ samples in the histopathological files of the Department of Pathology that had been surgically resected at the Department of Neurosurgery, Fukuoka University Hospital (Fukuoka, Japan), between January 1985 and December 2014 were reviewed. Tumor resection was performed to improve neurological symptoms and quality of life, and aimed to prolong the survival time of patients. Patients in which BM were resected included those in which: i) A single large $(>3 \mathrm{~cm}$ ) or symptomatic tumor was present; ii) the primary tumor was well-controlled; iii) life expectancy exceeded 3 months; and iv) written informed consent from the patient or family was obtained. A total of 68 patients with BM from lung adenocarcinoma were retrospectively analyzed. Analysis data from eight patients lacking clinical data and specimens, and from one patient in which polymerase chain reaction (PCR) amplification of EGFR failed were excluded. Data from a total of 59 patients were included in the present study. The current study was reviewed and approved by the Ethics Committee of the Fukuoka University Hospital (approval no. 15-5-14).

Pathologic evaluation. Surgically-resected specimens were fixed in $10 \%$ formalin (room temperature for $24 \mathrm{~h}$ ) and embedded in paraffin blocks. Tissue sections were cut at a thickness of $4 \mu \mathrm{m}$, and stained with hematoxylin and eosin for histopathological examination. Tissue sections were stained with hematoxylin for $10 \mathrm{~min}$, and with eosin $3 \mathrm{~min}$ at room temperature. Histological subtyping was performed according to the 2015 World Health Organization criteria for classification of lung tumors (18). The clinicopathological parameters considered in this study included patient age, sex, location of $\mathrm{BM}$, histological subtype of BM, smoking status, Karnofsky performance score (KPS), number of BM, presence of extracranial metastasis (ECM), GPA, timing of diagnosis, tyrosine kinase inhibitor (TKI) treatment prior to diagnosis of BM, presence of $R A S$ mutations or anaplastic lymphoma kinase $(A L K)$ rearrangements and re-operation.

Analysis of EGFR and KRAS mutations and ALK rearrangement. EGFR and $R A S$ gene mutations were analyzed in paraffin-embedded tissue sections from BM only. EGFR mutations (including G719X in exon 18, exon 19 deletion, T790M in exon 20, and L858R and L861Q in exon 21) and $R A S$ mutations (G12C and G12A in exon 2 and Q61H in exon 3) were detected in matching formalin-fixed paraffin-embedded tissue samples using cycleave PCR (which was performed by SRL Inc., Tokyo, Japan, cobas ${ }^{\circledR}$ EGFR Mutation Test v2 kit and MEBGEN ${ }^{\text {TM }}$ RASKET kit according to the manufacture's protocol). $A L K$ rearrangement was analyzed by immunohistochemistry (ALK Detection kit; Nichirei Bioscience, Tokyo, Japan), according to the manufacturer's protocols. The slides were observed under x200 magnification using a BX50 fluorescence microscope (Olympus Corporation, Tokyo, Japan).

Statistical analysis. To identify clinical features associated with EGFR mutations, categorical variables were compared using Wilcoxon signed-rank test or Fisher's exact test. OS was defined as duration between the date of BM diagnosis and the date of mortality or last follow-up. The Kaplan-Meier estimator method was used for survival curve analysis, and differences between survival curves were analyzed using the log-rank test. Multivariate analysis with the Cox proportional hazard model was used to determine independent prognostic factors. $\mathrm{P}<0.05$ were considered to indicate a statistically significant difference. Statistical analysis was performed with SPSS software (version 21.0; IBM Corp., Armonk, NY, USA).

\section{Results}

Patient characteristics. Patient characteristics are summarized in Table I. A total of 59 patients with BM from lung adenocarcinoma were included in the present study. The median patient age was $61.3 \pm 12.2$ years old (range, 38-87 years). A total of 45 patients were male $(76.3 \%)$ and 14 patients were female $(23.7 \%)$. A total of 14 patients (23.7\%) exhibited EGFR mutations (Table II), of which deletion of exon 19 was the most common genetic alteration (eight patients, 57.1\%), followed by L858R mutation in exon 21 (five patients, 35.7\%). In addition, one patient $(7.1 \%$ ) had a L861Q mutation in exon 21. EGFR mutations occurred significantly more often in female compared with male patients (64.3 vs. $35.7 \%$; $\mathrm{P}=0.0001)$. EGFR mutations also occurred significantly more frequently in non-smokers compared with current or ex-smokers (57.1 vs. 42.9\%; $\mathrm{P}=0.04)$. BM within the frontal lobe were significantly more common compared with other locations in patients with EGFR mutations [64.3 (frontal lobe) vs. 21.4 (cerebellum) vs. $7.1 \%$ (posterior lobe); $\mathrm{P}=0.03]$. The rate of surgery for tumor recurrence occurred significantly more often in patients with wild-type compared with mutated EGFR (37.8 vs. 7.1\%; $\mathrm{P}=0.04)$. The two groups did not significantly differ in GPA measures of age, KPS, number of BM, presence of ECMs, timing of diagnosis, and frequency of $R A S$ mutation and $A L K$ rearrangements.

All patients received craniotomy followed by total resection of the BM. In 32 patients (54.2\%), ECMs were detected at the time of BM diagnosis. The sites at which ECMs occurred in the patients with EGFR mutation were similar to those in which ECMs occurred in patients with WT. In patients with EGFR mutations, six patients had ECMs (one patient had multiple lesions). Of these ECMs, six occurred in the lymph nodes $(100 \%)$, one occurred in bone $(16.7 \%)$, and one occurred 
Table I. Patient baseline characteristics $(n=59)$.

\begin{tabular}{|c|c|c|c|c|}
\hline Characteristic & Total $(n=59)$ & EGFR MT $(n=14)$ & WT $(n=45)$ & P-value MT vs. WT \\
\hline Age, years ${ }^{\mathrm{a}}$ & $61.3 \pm 12.2$ & $61.6 \pm 12.0$ & $61.1 \pm 12.4$ & 0.80 \\
\hline $\operatorname{Sex}(\%)$ & & & & 0.0001 \\
\hline Male & $45(76.3)$ & $5(35.7)$ & $40(88.9)$ & \\
\hline Female & $14(23.7)$ & $9(64.3)$ & $5(11.1)$ & \\
\hline \multicolumn{5}{|l|}{ Location $(\%)$} \\
\hline Frontal lobe & $23(39.0)$ & $9(64.3)$ & $14(31.1)$ & 0.03 \\
\hline Cerebellum & $11(18.6)$ & $3(21.4)$ & $8(17.8)$ & 0.76 \\
\hline Posterior lobe & $8(15.6)$ & $1(7.1)$ & 7 (15.6) & 0.67 \\
\hline \multicolumn{5}{|l|}{ Histological subtype (\%) } \\
\hline Solid & $34(57.6)$ & $4(28.6)$ & $30(66.7)$ & 0.03 \\
\hline Papillary & $13(22.0)$ & $5(35.7)$ & $8(17.7)$ & 0.26 \\
\hline Acinar & $11(18.6)$ & $4(28.6)$ & 7 (15.6) & 0.43 \\
\hline Smoking status (\%) & & & & 0.04 \\
\hline Never & $19(32.2)$ & $8(57.1)$ & $11(24.4)$ & \\
\hline Current or past & $40(67.8)$ & $6(42.9)$ & $34(75.6)$ & \\
\hline \multicolumn{5}{|l|}{ KPS (\%) } \\
\hline$<70$ & $19(32.2)$ & $6(42.9)$ & $13(28.9)$ & 0.51 \\
\hline $70-80$ & $15(25.4)$ & $5(35.7)$ & $10(22.2)$ & 0.48 \\
\hline $81-100$ & $25(42.4)$ & $3(21.4)$ & $22(48.9)$ & 0.12 \\
\hline \multicolumn{5}{|l|}{ Number of BM (\%) } \\
\hline 1 & $31(52.5)$ & $5(35.7)$ & $26(45.8)$ & 0.22 \\
\hline $2-3$ & $17(28.8)$ & $5(35.7)$ & $12(26.7)$ & 0.74 \\
\hline$>3$ & $11(18.6)$ & $4(28.6)$ & $7(15.6)$ & 0.43 \\
\hline $\mathrm{ECM}(\%)$ & & & & 0.80 \\
\hline Absent & $27(45.8)$ & $6(42.9)$ & $21(46.7)$ & \\
\hline Present & $32(54.2)$ & $8(57.1)$ & $24(53.3)$ & \\
\hline \multicolumn{5}{|l|}{$\operatorname{GPA}(\%)$} \\
\hline $0-1.0$ & $13(22.0)$ & $4(28.6)$ & $9(20.0)$ & 0.71 \\
\hline $1.5-2.0$ & $24(40.7)$ & $7(50.0)$ & $17(37.8)$ & 0.54 \\
\hline $2.5-3.0$ & $18(30.5)$ & $3(21.4)$ & $15(33.3)$ & 0.52 \\
\hline $3.5-4.0$ & $4(6.8)$ & $0(.0)$ & $4(8.9)$ & 0.56 \\
\hline \multicolumn{5}{|l|}{ Timing of diagnosis } \\
\hline $\begin{array}{l}\text { BM diagnosed before } \\
\text { PT }(\%)\end{array}$ & $27(45.8)$ & $5(35.7)$ & $22(48.8)$ & 0.54 \\
\hline TKI (prior to BM) (\%) & $2(3.4)$ & $2(14.3)$ & $0(.0)$ & 0.05 \\
\hline RAS (\%) & $5(8.5)$ & $0(.0)$ & $5(11.1)$ & 0.16 \\
\hline ALK $(\%)$ & $3(5.0)$ & $0(.0)$ & $3(6.7)$ & 0.57 \\
\hline Re-operation (\%) & $18(30.5)$ & $1(7.1)$ & $17(37.8)$ & 0.04 \\
\hline
\end{tabular}

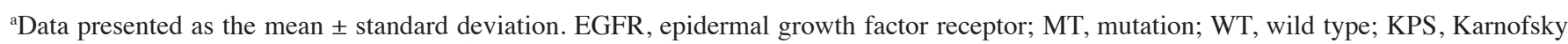
performance score; BM, brain metastasis; ECM, extracranial metastasis; GPA, graded prognostic assessment; PT, primary tumor; TKI, tyrosine kinase inhibitor; ALK, anaplastic lymphoma kinase.

in liver (16.7\%). In patients with WT, 21 patients had ECMs (4 patients had multiple lesions). Of these lesions, 15 occurred in the lymph nodes (71.4\%), six occurred in bone (28.6\%), two occurred in liver $(9.5 \%)$ and two occurred in the adrenal glands $(9.5 \%)$. There was no statistically significant difference in the sites at which ECMs occurred in comparing patients with these two conditions. Regarding the number of BM present, one in 31 patients $(52.5 \%)$, two in 13 patients $(22.0 \%)$, three in four patients $(6.8 \%)$, four in two patients $(3.4 \%)$ and $\geq$ five in nine patients $(15.3 \%)$. Following surgical resection of BM, the majority of patients received local therapy (38 patients, 64.4\%), consisting of whole brain radiation therapy (WBRT; 24 patients, 
Table II. EGFR mutation analysis with brain metastasis from lung adenocarcinoma $(n=59)$.

\begin{tabular}{lc}
\hline EGFR mutation & No. of patients $(\%)$ \\
\hline Exon 19 deletion & $8(57.1)$ \\
Exon 21 L858R & $5(35.7)$ \\
Exon 21 L861Q & $1(7.1)$ \\
Exon 18 G719X & $0(0.0)$ \\
Exon 20 T790M & $0(0.0)$ \\
\hline
\end{tabular}

A total of $14 / 59$ patients had EGFR mutations. EGFR, epidermal growth factor receptor.

$40.7 \%$ ) or stereotactic radiosurgery (SRS; 14 patients, $23.7 \%$ ). Following local therapy, 18 patients $(30.5 \%)$ received systemic chemotherapy. A total of five patients $(8.5 \%)$ received systemic chemotherapy only, while two patients $(3.4 \%)$ with EGFR mutations were treated with the EGFR-TKI gefitinib prior to the diagnosis of BM. Patients with poor performance status and patients that declined treatment (14 patients, 23.7\%) were provided with best supportive care.

Pathological subtypes. The predominant subtypes of BM associated with lung adenocarcinoma were solid adenocarcinoma (34 patients, $57.6 \%$ ), followed by papillary adenocarcinoma (13 patients, 22.0\%), acinar adenocarcinoma (11 patients, $18.6 \%$ ) and micropapillary adenocarcinoma (one patient, $1.8 \%$ ) (Table I). EGFR mutations occurred most commonly in papillary adenocarcinoma (five patients, 35.7\%), followed by acinar adenocarcinoma (four patients, 26.7\%), solid adenocarcinoma (four patients, 26.7\%) and micropapillary adenocarcinoma (one patient, 7.1\%). No significant association was identified between the occurrence of an EGFR mutation and histological subtype. However, the wild-type EGFR gene was significantly more common in patients with solid adenocarcinoma compared with other histological subtypes. Fig. 1 shows representative histological subtypes of BM from lung adenocarcinoma.

Tissue from the primary lung adenocarcinoma together with BM were available for 20 patients. Characteristics of primary lung adenocarcinoma and BM are summarized in Table III. The most common histological subtype in patients with primary lung adenocarcinoma who also had BM was solid adenocarcinoma (10 patients, $50.0 \%$ ), followed by acinar adenocarcinoma (seven patients, 35.0\%), and papillary adenocarcinoma (three patients, $15.0 \%$ ). Histopathological subtypes of lung adenocarcinoma and BM matched each other in $75 \%$ of all of cases.

Patient survival. After a mean follow-up duration of 17.9 months, 39/59 patients $(66.1 \%)$ had succumbed, 10 patients $(16.9 \%)$ survived and 10 patients (16.9\%) were lost to follow-up. The median OS was 19.2 months. Table IV presents the results of the OS univariate analysis. The OS time was significantly increased in patients with EGFR mutations (median OS of 31.5 months vs. 15.6 months; $\mathrm{P}=0.02$; Fig. 2A). No significant difference was observed in OS in patients with exon 19 deletion compared with those with L858R mutations in exon 21 ( $\mathrm{P}=0.31$, data not shown). In addition, the OS time was significantly increased in patients with $\mathrm{BM}$ in the frontal lobe compared with patients with BM the cerebellum or in other regions [median OS, 28.2 (BM in the frontal lobe) vs. 13.7 (BM in the cerebellum) vs. 12.7 months (BM in other brain regions); $\mathrm{P}=0.004$ ] (Fig. 2B) and in non-smokers (33.1 months in non-smokers vs. 12.5 months in current or ex-smokers; $\mathrm{P}=0.001$; Fig. 2C).

Patients diagnosed with BM following initial diagnosis of the primary lung adenocarcinoma primarily had a longer OS, compared with patients with an initial diagnosis of BM prior to lung cancer (median OS, 23.4 vs. 12.5 months; $\mathrm{P}=0.079$ ), but this did not reach statistical significance. Patient age, histological subtype of BM, KPS and re-operation were not identified to be associated with OS. Statistical analysis of patients with $A L K$ rearrangement was not feasible as only three patients with this genetic change were present in the patient population.

Multivariate analysis suggested that BM located in the frontal lobe and a non-smoker status were independent and significant prognostic factors $(\mathrm{P}=0.007$ and $\mathrm{P}=0.008$, respectively; Table V).

\section{Discussion}

In the present study, the clinical and histological features of patients that developed BM from lung adenocarcinoma were evaluated in order to identify prognostic factors for such patients. Previous studies have investigated the incidence of EGFR mutations in a large population of patients with lung cancer $(19,20)$. EGFR mutations have been detected in $30 \%$ of patients with adenocarcinoma, $38 \%$ of female patients, $47 \%$ of non-smokers and $32 \%$ of patients of East Asian origin in the patients with lung adenocarcinoma $(19,21)$. It has also been reported to be easier to detect EGFR mutations in smaller primary tumors (22). Deletion of exon 19 and L858R mutations in exon 21 account for $\sim 85 \%$ of EGFR mutations and have been recognized as important markers in determining the appropriate treatment strategy for patients with advanced lung cancer (21). BM from lung cancer were detected at the time of diagnosis of the primary tumor in $8-23 \%$ of patients with EGFR mutations, and in $24 \%$ of patients with NSCLC during follow-up care $(23,24)$. In patients with NSCLC in which EGFR mutations were present, $34.2 \%$ were identified to have developed BM 1 year after diagnosis, $38.4 \%$ at 2 years, $46.7 \%$ at 3 years, $48.7 \%$ at 4 years and $52.9 \%$ at 5 years in the USA (25).

At the time of the initial diagnosis of primary lung adenocarcinoma, the incidence of EGFR mutation is reported to vary according to the site of BM $(24,26)$. Patients with lung adenocarcinoma with EGFR mutations have been suggested to have a higher incidence rate of BM compared with patients without mutations $(24,26)$. However, another study reported that the timeline of development of BM was not significantly longer in EGFR-mutated NSCLC compared with wild-type EGFR NSCLC (27). Therefore, the association between the presence of EGFR mutations and BM is controversial. Multiple BM $(\geq 2)$ have been reported to occur more frequently in patients with EGFR mutations, but the size of the largest 
Table III. Pathological subtype of primary lung adenocarcinoma and metastatic brain tumor $(\mathrm{n}=20)$.

\begin{tabular}{|c|c|c|c|c|}
\hline No. & Stage & Size $(\mathrm{mm})$ & Primary & $\mathrm{BM}$ \\
\hline 1 & T1aN0Mx & $11 \times 10 \times 15$ & Solid (solid + acinar) & Solid \\
\hline 2 & T1bN0M0 & $20 \times 15 \times 20$ & Solid (solid + acinar) & Acinar \\
\hline 3 & $\mathrm{~T} 2 \mathrm{aNxMx}$ & $40 \times 30 \times 25$ & Acinar & Acinar \\
\hline 4 & T1cN0M0 & $28 \times 26 \times 24$ & Acinar (acinar + solid $)$ & Acinar \\
\hline 5 & T1bN0Mx & $20 \times 15 \times 15$ & Acinar (acinar + papillary) & Papillary \\
\hline 6 & T1bNxMx & $20 \times 10 \times 8$ & Solid & Solid \\
\hline 7 & T1bNxMx & $20 \times 8 \times 8$ & Solid (acinar + papillary + MPP) & Solid \\
\hline 8 & $\mathrm{~T} 2 \mathrm{aNxMx}$ & $30 \times 38 \times 35$ & Solid (solid + acinar + papillary) & Acinar \\
\hline 9 & T4N2M1 & $40 \times 35 \times 35$ & Acinar (acinar + MPP) & Solid \\
\hline 10 & T1cN0Mx & $30 \times 25 \times 20$ & Solid (solid + acinar) & Solid \\
\hline 11 & $\mathrm{~T} 2 \mathrm{NxM} 1$ & $15 \times 10 \times 10$ & Solid (solid + acinar + MPP) & Solid \\
\hline 12 & T3N0M1 & $40 \times 35 \times 35$ & Solid (solid + acinar) & Solid \\
\hline 13 & T4N0Mx & $70 \times 70 \times 55$ & MPP (MPP + acinar + solid) & Solid \\
\hline 14 & T1bN0Mx & $16 \times 15 \times 15$ & Solid (solid + acinar) & Papillary \\
\hline 15 & $\mathrm{~T} 2 \mathrm{aN} 2 \mathrm{M} 0$ & $18 \times 17 \times 24$ & MPP (MPP + acinar + papillary) & Solid \\
\hline 16 & $\mathrm{~T} 4 \mathrm{~N} 2 \mathrm{Mx}$ & $85 \times 60 \times 50$ & Solid (solid + MPP + acinar) & Solid \\
\hline 17 & $\mathrm{~T} 1 \mathrm{cNxMx}$ & $22 \times 18 \times 10$ & Acinar (acinar + MPP + papillary) & MPP \\
\hline 18 & $\mathrm{~T} 2 \mathrm{bN} 2 \mathrm{Mx}$ & $50 \times 50 \times 30$ & Acinar (acinar + solid + papillary $)$ & Solid \\
\hline 19 & $\mathrm{~T} 1 \mathrm{cNxMx}$ & $30 \times 20 \times 27$ & Acinar (acinar + papillary) & Papillary \\
\hline 20 & $\mathrm{~T} 2 \mathrm{aNxMx}$ & $35 \times 40 \times 25$ & MPP (MPP + acinar) & Papillary \\
\hline
\end{tabular}

BM, brain metastasis; MPP, micropapillary.

A

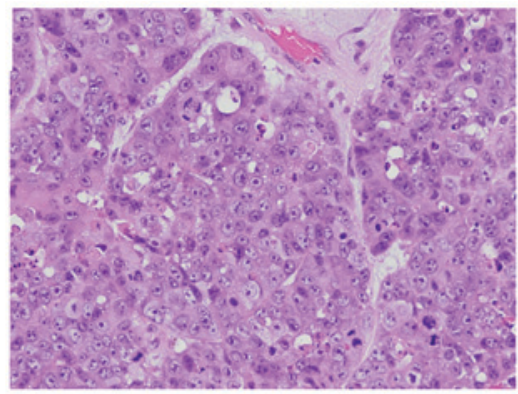

$\mathrm{C}$

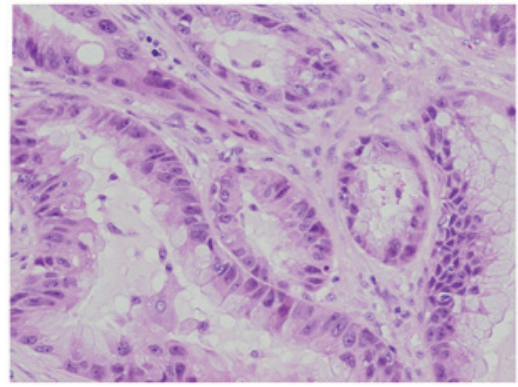

B

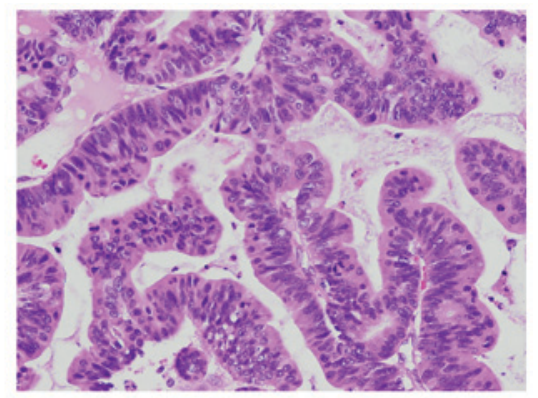

D

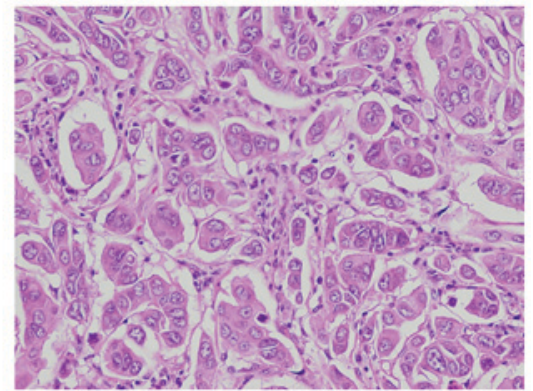

Figure 1. Histopathological subtypes of brain metastases from lung adenocarcinoma (hematoxylin and eosin staining; magnification, x200). (A) Solid predominant, (B) papillary predominant, (C) acinar predominant and (D) micropapillary predominant.

BM does not appear to be associated with the presence of EGFR mutations (28).

Several studies have compared EGFR status in the primary tumor and BM in patients with $\operatorname{NSCLC~}(26,29)$. These results suggested high concordance rates in EGFR mutation status $(93.3-100 \%)$ between the primary tumor and BM $(26,29)$. In the current study, 5/20 patients examined had EGFR mutations in the primary lung adenocarcinoma and BM. Five patients had the same EGFR mutation in both sites (three patients had exon 19 deletions and one patient each had had L858R and L861Q substitutions in exon 21). In the present study, the frequency of EGFR mutations in patients with BM (23.7\%) was 

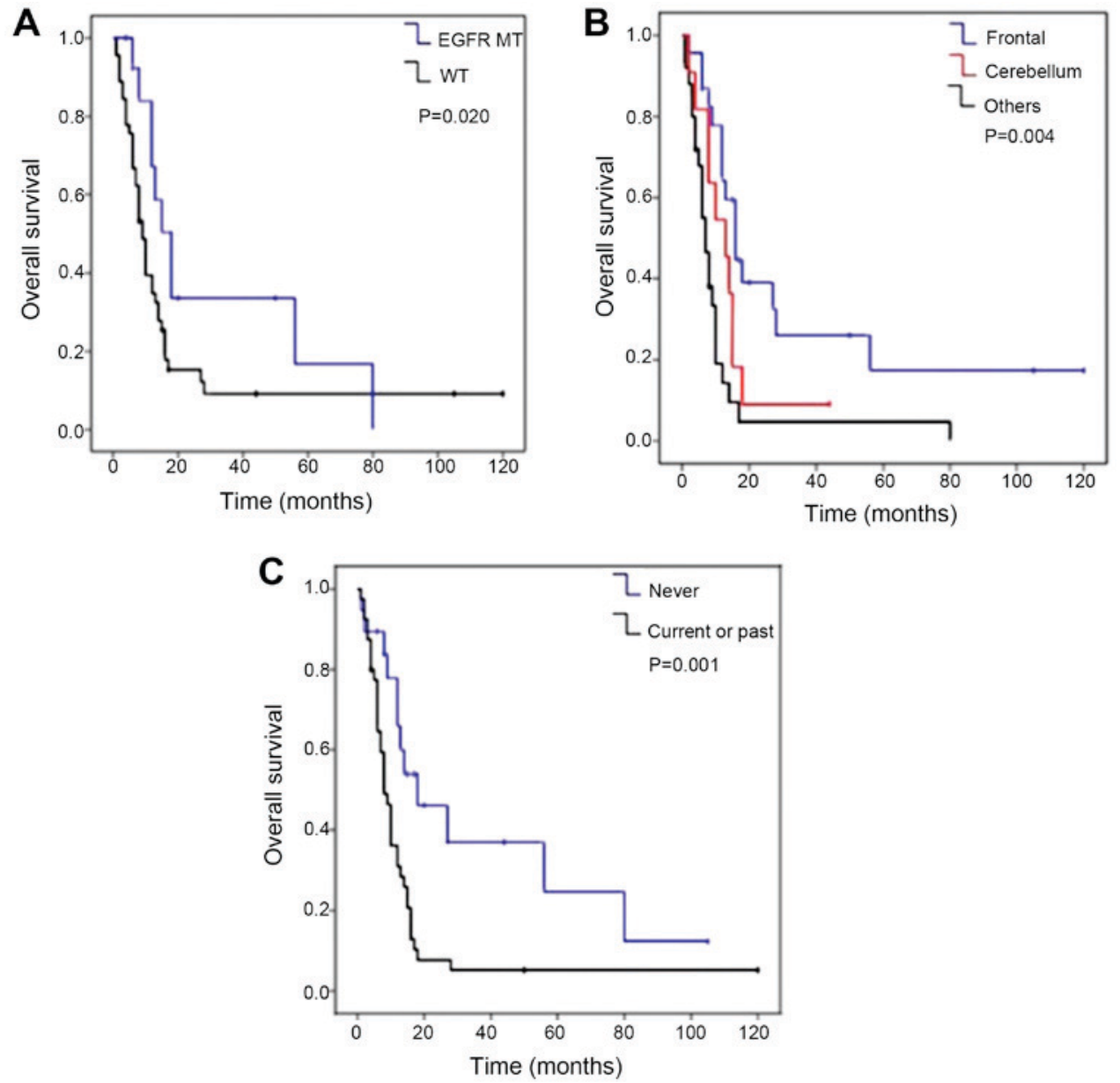

Figure 2. Kaplan-Meier curves of OS. Curves of OS were analyzed in accordance with (A) EGFR mutation status, (B) location of BM and (C) smoking. BM, brain metastases; EGFR, epidermal growth factor receptor; MT, mutation; WT, wild type; OS, overall survival.

lower compared with previously reported $(19,21)$. This discrepancy may be due to the characteristics of the patient population in the current study. EGFR mutations in lung adenocarcinoma are most common in female patients and non-smokers, but the present study cohort included predominantly male patients (76.3\% of all patients).

The International Association for the Study of Lung Cancer/American Thoracic Society/European Respiratory Society criteria for lung adenocarcinoma classification (30), and the 2015 WHO criteria lung tumor classification (18) recommend the identification of pathological patterns. Primary invasive lung adenocarcinoma has five architectural growth patterns, including lepidic, acinar, papillary, solid and micropapillary patterns. Tsuta et al (31) reported that the most prevalent subtype of adenocarcinoma was papillary-predominant (37.1\%), followed by lepidic-predominant $(15.1 \%)$, solid with mucin production-predominant (13.7\%), acinar-predominant $(10.8 \%)$ and micropapillary-predominant $(6.7 \%)$ in a study of 757 cases of invasive lung adenocarcinoma (31). In a previous study of 119 patients with primary lung adenocarcinoma, the most common histopathological subtype reported was papillary-predominant (58\%), followed by acinar-predominant (16\%), micropapillary-predominant (9\%), solid-predominant $(4 \%)$ and lepidic-predominant (2\%) (32). EGFR mutation have been reported to occur significantly more often in adenocarcinoma papillary predominant, compared with all other subtypes (31).
To the best of our knowledge, only one report has demonstrated the clinical relevance of pathological subtypes in metastatic lung adenocarcinoma (16). In this study of 100 patients with metastatic lung adenocarcinoma, the most frequent histological pattern was solid with mucin $(50 \%)$, followed by acinar (29\%), micropapillary (20\%) and papillary (1\%). This study included 30 tissue samples from BM. The study authors hypothesized that this difference in predominant components in primary and metastatic sites was based on the function of the metastatic potential of each adenocarcinoma subtype. The major solid histopathological subtype of metastatic lung adenocarcinoma was associated with poorer OS compared with other histopathological subtypes (16). Furthermore, this histopathological subtype was less likely to harbor EGFR mutations and occurred less frequently in never smokers (16). In a separate study, Amin et al (17) reported micropapillary predominant adenocarcinoma may be more likely to metastasize. The present study also identified that the most frequent subtype of BM from lung adenocarcinoma was solid predominant (57.6\%). OS did not vary as a function of histological subtype. However, wild type EGFR was significantly more common in patients with the solid predominant subtype. This data is consistent with previous reports $(30,31)$, but these results require validation in larger cohorts.

In recent years, TKIs targeting mutated EGFR proteins have been established as the standard treatment for NSCLC. 
Table IV. Univariate analysis of overall survival with brain metastasis from lung adenocarcinoma.

\begin{tabular}{|c|c|c|c|}
\hline Variable & No. of patients & Median OS (m) & P-value \\
\hline Age & & & 0.729 \\
\hline$<70$ & 45 & $22.7(11.8-33.7)$ & \\
\hline$\geqq 70$ & 14 & $19.4(9.1-29.7)$ & \\
\hline Sex & & & 0.131 \\
\hline Male & 45 & $16.9(11.4-22.4)$ & \\
\hline Female & 14 & $27.8(15.7-39.9)$ & \\
\hline Location & & & 0.004 \\
\hline Frontal & 23 & $28.2(19.0-37.5)$ & \\
\hline Cerebellum & 11 & $13.7(7.4-20.0)$ & \\
\hline Others & 25 & $12.7(5.9-19.5)$ & \\
\hline Smoking & & & 0.001 \\
\hline Never & 19 & $33.1(22.1-33.1)$ & \\
\hline Current or past & 40 & $12.5(8.3-16.7)$ & \\
\hline Histological subtype & & & 0.236 \\
\hline Solid & 34 & $17.4(11.4-23.5)$ & \\
\hline Papillary & 13 & $26.9(13.0-40.8)$ & \\
\hline Aciner & 11 & $11.2(5.9-16.6)$ & \\
\hline EGFR & & & 0.020 \\
\hline Mutation & 14 & $31.5(19.3-43.8)$ & \\
\hline Wild type & 45 & $15.6(10.3-20.8)$ & \\
\hline KPS & & & 0.551 \\
\hline$<70$ & 19 & $18.2(8.8-27.6)$ & \\
\hline $70-80$ & 15 & $17.1(8.3-25.9)$ & \\
\hline $81-100$ & 25 & $21.9(13.4-30.4)$ & \\
\hline GPA & & & 0.180 \\
\hline $0-1.0$ & 13 & $22.8(10.0-35.6)$ & \\
\hline $1.5-2.0$ & 24 & $12.8(7.8-17.8)$ & \\
\hline $2.5-3.0$ & 18 & $20.2(11.5-28.8)$ & \\
\hline $3.5-4.0$ & 4 & $37.0(12.7-61.2)$ & \\
\hline Timing of diagnosis & & & 0.079 \\
\hline \multicolumn{4}{|c|}{$\mathrm{BM}$ diagnosed before PT } \\
\hline Yes & 27 & $12.5(8.3-16.9)$ & \\
\hline No & 32 & $23.4(15.6-31.3)$ & \\
\hline RAS & & & 0.074 \\
\hline Mutation & 5 & $7.8(4.7-10.8)$ & \\
\hline Wild type & 54 & $23.7(13.8-33.5)$ & \\
\hline Re-operation & & & 0.712 \\
\hline Present & 18 & $17.7(8.8-26.7)$ & \\
\hline Absent & 41 & $19.7(13.3-26.1)$ & \\
\hline
\end{tabular}

EGFR, epidermal growth factor receptor; OS, overall survival; KPS, Karnofsky performance score; GPA, graded prognostic assessment; BM, brain metastasis; PT, primary tumor.

This treatment is associated with longer progression-free survival and OS compared with treatment with conventional chemotherapy $(11,33)$, particularly in certain populations, including Eastern Asian, female and non-smoker patients with adenocarcinoma (14). In addition to demonstrating improved efficacy, TKI treatment is associated with less toxicity relative to conventional chemotherapy. Several studies have reported that the TKI treatment of patients with NSCLC with BM results in intracranial response rates ranging between 26.7 and $83 \%(14,15)$, and rates of $64-87 \%$ when combined with WBRT (34). TKI treatment for the patients with BM resulted in reduced tumor size and significantly improved OS (18.8 
Table V. Multivariate analysis of overall survival with brain metastasis of lung adenocarcinoma.

\begin{tabular}{lccc}
\hline Variable & HR & $95 \%$ CI & P-value \\
\hline Location, frontal & 0.552 & $0.215-0.781$ & 0.007 \\
Never smoking & 2.654 & $1.295-5.442$ & 0.008 \\
EGFR mutation & 0.552 & $0.260-1.171$ & 0.121 \\
\hline
\end{tabular}

EGFR, epidermal growth factor receptor; HR, hazard ratio; CI, confidence interval.

vs. 11.1 months) (13-15). In the present study, only two patients received TKI treatment prior to surgical resection, as the majority of patients were treated prior to the development of TKIs. Excluding these two patients, there was no statistically significant difference in OS between patients with and without EGFR mutations (17.1 vs. 15.6 months). The presence of an EGFR mutation prolonged OS, but improved outcome may be due to the efficacy of treatment with TKIs. In agreement with the current results, for patients with BM from lung adenocarcinoma, the presence of EGFR mutations has been associated with improved survival from the time of development of BM (15-25 vs. 7 months) (35-37). However, no significant difference was observed in the timeline of BM development in patients treated with EGFR-TKI compared with those receiving conventional chemotherapy (24).

To the best of our knowledge, no previous study has reported an association between EGFR mutation and the site of BM. Thus, the findings in the present study of a significant association between EGFR mutations and BM in the frontal lobe is novel. In low-grade gliomas, lesions localized in the frontal lobe may be vigorously resected without causing persistent functional deficits (38). The frontal lobe, which does not contain vital brain regions controlling speech and motor areas, is a brain region in which relatively aggressive surgical treatment may be pursued with lower risk of causing lasting and serious functional deficits.

In patients with BM, RPA and GPA scores are still the most widely accepted variables considered in evaluating disease prognosis $(6,9)$. However, the present study indicated that the presence of EGFR mutations, BM within the frontal lobe and non-smoker status are additional independent prognostic factors.

A long time period was investigated for retrospective analysis to evaluate the clinicopathological and genetic changes of BM from lung adenocarcinoma. The diagnostic neuroimaging and the treatment modalities, including WRRT, SRS and chemotherapy have been developed. Nonetheless, these results remain valid. The most common histological subtype in patients with primary lung adenocarcinoma was the papillary subtype. However, the predominant subtype of BM from lung adenocarcinoma was solid adenocarcinoma in this study. The clinicopathological characteristics of patients are not affected by the development of treatment. While the interval between detection of the primary cancer and detection of BM increased over the period covered in this study, the interval elapsed between detection of BM and the date of patient mortality or last follow-up did not change significantly.
Therefore, the prognostic factors identified in this study are reliable.

The limitations of the current study include its retrospective nature, and the potential bias in treatment strategy following resection of BM cannot be excluded. Furthermore, other clinically important genetic changes, including $A L K$ rearrangement, were not assessed due to the limited number of patients with this genetic alteration in the present cohort. Despite these limitations, to the best of our knowledge, this is the first report to investigate the histopathological subtypes of $\mathrm{BM}$ from lung adenocarcinoma, and the association between these subtypes, EGFR mutations and disease prognosis in a large patient cohort.

In the present study, univariate analysis was used to demonstrated that three factors, EGFR mutation, BM localized to the frontal lobes and non-smoker status, may be used as prognostic indicators. In addition, multivariate analysis confirmed these results, and demonstrated that the presence of $\mathrm{BM}$ in the frontal lobes and non-smoker status were the most informative prognostic factors. However, these conclusions are primarily applicable to postoperative assessment of $\mathrm{BM}$. The present study suggests that tissue from the BM may be useful as an alternative tissue for analysis, as resectable $\mathrm{BM}$ from lung adenocarcinoma cases exhibit characteristics that are similar to those of the primary tumor tissue. TKI treatment for BM from lung adenocarcinoma should be considered if indicated by histopathological and genetic examination.

\section{Acknowledgements}

The authors would like to thank Ms. M. Onitsuka and Ms. H. Fukagawa from the Department of Pathology, Faculty of Medicine, Fukuoka University (Fukuoka, Japan) for excellent technical assistance with immunohistochemistry.

\section{Funding}

The present study was supported in part by a grant from the Research Center for Advanced Molecular Medicine, Fukuoka University, Fukuoka, Japan.

\section{Availability of data and materials}

All data generated and/or analyzed during this study are included in this published article.

\section{Authors' contributions}

$\mathrm{HK}$ and $\mathrm{MH}$ analyzed statistics, and drafted the manuscript. HK, MH, MY HA and MN analyzed and interpreted the patient data. TM TI and KN contributed to the conception of the study, critically reviewed the manuscript and supervised the study. All authors read and approved the final manuscript.

\section{Ethics approval and consent to participate}

Written informed consent was obtained, and the study design was approved by our institutional review board (IRB protocol \#15-5-14). 


\section{Patient consent for publication}

Patients provided written informed consent for the publication of any data and associated images.

\section{Competing interests}

The authors declare that they have no competing interests.

\section{References}

1. Shi AA, Digumarthy SR, Temel JS, Halpern EF, Kuester LB and Aquino SL: Does initial staging or tumor histology better identify asymptomatic brain metastases in patients with non-small cell lung cancer? J Thorac Oncol 1: 205-210, 2006.

2. Heon S, Yeap BY, Britt GJ, Costa DB, Rabin MS, Jackman DM and Johnson BE: Development of central nervous system metastases in patients with advanced non-small cell lung cancer and somatic EGFR mutations treated with gefitinib or erlotinib. Clin Cancer Res 16: 5873-5882, 2010.

3. Barnholtz-Sloan JS, Sloan AE, Davis FG, Vigneau FD, Lai P and SAwaya RE: Incidence proportions of brain metastases in patients diagnosed (1973 to 2001) in the metropolitan detroit cancer surveillance system. J Clin Oncol 22: 2865-2872, 2004.

4. Cox JD, Scott CB, Byhardt RW, Emami B, Russell AH, Fu KK, Parliament MB, Komaki R and Gasper LE: Addition of chemotherapy to radiation therapy alters failure patterns by cell type within non-small cell carcinoma of lung (NSCCL): Analysis of radiation therapy oncology group (RTOG) trials. Int Radiat Oncol Biol Phys 43: 505-509, 1999.

5. Wang SY, Ye X, Ou W, Lin YB, Zhang BB and Yang H: Risk of cerebral metastases for postoperative locally advanced non-small-cell lung cancer. Lung Cancer 64: 238-243; 2009.

6. Gaspar L, Scott C, Rotman M, Asbell S, Phillips T, Wasserman T, McKenna WG and Byhardt R: Recursive partitioning analysis (RPA) of prognostic factors in three radiation therapy oncology group (RTOG) brain metastases trials. Int J Radiat Oncol Biol Phys 37: 745-751, 1997.

7. Sperduto PW, Berkey B, Gasper LE, Mehta M and Curran W: A new prognostic index and comparison to three other indices for patients with brain metastases: An anlysis of 1,960 patients in the RTOG database. Int J Radiat Oncol Biol Phys 70: 510-514, 2008 .

8. Chang DB, Yang PC, Luh KT, Kuo SH, Hong RL and Lee LN: Late survival of non-small cell lung cancer patients with brain metastases. Influence of treatment. Chest 101: 1293-1297, 1992.

9. Kepka L, Cieslak E, Bujko K, Fijuth J and Wierzchowski M: Results of the whole-brain radiotherapy for patients with brain metastases from lung cancer: The RTOG RPA intra-classes analysis. Acta Oncol 44: 389-398, 2005

10. Paez JG, Jänne PA, Lee JC, Tracy S, Greulich H, Gabriel S, Herman P, Kaye FJ, Lindeman N, Boggon TJ, et al: EGFR mutations in lung cancer: Correlation with clinical response to gefitinib therapy. Science 304: 1497-1500, 2004

11. Mok TS, Wu YL, Thongprasert S, Yang CH, Chu DT, Saijo N, Sunpaweravong P, Han B, Margono B, Ichinose Y, et al: Gefitinib or carbonplatin-paclitaxel in pulmonary adenocarcinoma. $\mathrm{N}$ Engl J Med 361: 947-957, 2009.

12. Maemondo M, Inoue A, Kobayashi K, Sugawara S, Oisumi S, Isobe $\mathrm{H}$, Gemma A, Harada M, Yoshizawa H, Kinoshita I, et al: Gefitinib or chemotherapy for non-small-cell lung cancer with mutated EGFR. N Engl J Med 362: 2380-2388, 2010.

13. Shimato S, Mitsudomi T, Kosaka T, Yutabe Y, Wakabayashi T, Mizuno M, Nakahara N, Hatano H, Natsume A, Ishii D and Yoshida J: EGFR mutations in patients with brain metastases from lung cancer: Association with the efficacy of gefitinib. Neuro Oncol 8: 137-144, 2006.

14. Kim JE, Lee DH, Choi Y, Yoon DH, Kim SW, Suh C and Lee JS: Epidermal growth factor receptor tyrosine kinase inhibitors as a first-line therapy for never-smokers with adenocarcinoma of the lung having asymptomatic synchronous brain metastasis. Lung Cancer 65: 351-354, 2009.

15. Park SJ, Kim HT, Lee DH, Kim KP, Kim SW, Suh C and Lee JS Efficacy of epidermal growth factor receptor tyrosine kinase inhibitors for brain metastasis in non-small cell lung cancer patients harboring either exon 19 or 21 mutation. Lung Cancer 77 : 556-560, 2012.
16. Clay TD, Do H, Sundararajan V, Moore MM, Conron M, Wright GM, McLachlan SA, Dobrovic A and Russell PA: The clinical relevance of pathologic subtypes in metastatic lung adenocarcinoma. J Thorac Oncol 9: 654-663, 2014

17. Amin MB, Tamboli P, Merchant SH, Ordonez NG, Ro J, Ayala AG and Ro JY: Micropapillary component in lung adenocarcinoma: A distinctive histologic feature with possible prognostic significance. Am J Surg Pathol 26: 358-364, 2002.

18. Travis WD BE, Burke AP, Marx A and Nicholson AG: WHO Classification of Tumors of the Lung, Pleura, Thymus and Heart. 4th edition. IARC press, Lyon, 2015.

19. Mitsudomi T and Yatabe Y: Mutations of the epidermal growth factor receptor gene and related genes as determinants of epidermal growth factor receptor tyrosine kinase inhibitors sensitivity in lung cancer. Cancer Sci 98: 1817-1824, 2007.

20. Mitsudori T, Kosaka T and Yatabe Y: Biological and clinical implications of EGFR mutations in lung cancer. Int $\mathrm{J}$ Clin Oncol 11: 190-198, 2006.

21. Shigematsu H, Lin L, Takahasi T, Nomura M, Suzuki M, Wistuba II, Fong KM, Lee H, Toyooka S, Shimizu N, et al: Clinical and biological features associated with epidermal growth factor receptor gene mutations in lung cancers. J Natl Cancer Inst 97: 339-346, 2005.

22. Yano M, Sasaki H, Kobayashi Y, Yukiue H, Haneda H, Suzuki E, Endo K, Kawano O, Hara M and Fujii Y: Epidermal growth factor receptor gene mutation and computed tomographic findings in peripheral pulmonary adenocarcinoma. J Thorac Oncol 1: 413-416, 2006.

23. Doebele RC, Lu X, Sumey C, Maxson DA, Weickhardt AJ, Oton AB, Bunn PA Jr, Barón AE, Franklin WA, Aisner DL, et al: Oncogene status predicts patterns of metastatic spread in treatment-naïve nonsmall cell lung cancer. Cancer 118: 4502-4511, 2012.

24. Hendriks LE, Smit EF, Vosse BA, Mellema WW, Heideman DA, Bootsma GP, Westenend M, Pitz C, de Vries GJ, Houben R, et al: EGFR mutated non-small cell lung cancer patients: More prone to development of bone and brain metastases? Lung Cancer 84: 86-91, 2014.

25. Rangachari D, Yamaguchi N, VanderLaan PA, Folch E, Mahadevan A, Floyd SR, Uhlmann EJ, Wong ET, Dahlberg SE, Huberman MS and Costa DB: Brain metastases in patients with EGFR-mutated or ALK-rearranged non-small-cell lung cancers. Lung Cancer 88: 108-111, 2015.

26. Matsumoto S, Takahasi K, Iwakawa R, Matsuno Y, Nakanishi Y, Kohno T, Shimizu E and Yokota J: Frequent EGFR mutations in brain metastases of lung adenocarcinoma. Int J Cancer 119: 1491-1494, 2006

27. Eichler AF, Kahle KT, Wang DL, Joshi VA, Willers H, Engelman JA, Lynch TJ and Sequist LV: EGFR mutation status and survival after diagnosis of brain metastasis in nonsmall cell lung cancer. Neuro Oncol 12: 1193-1199, 2010.

28. Shin DY, Na II, Kim CH, Park S, Baek H and Yang SH: EGFR mutation and brain metastasis in pulmonary adenocarcinomas. J Thorac Oncol 9: 195-199, 2014

29. Sun M, Behrens C, Feng L, Ozburn N, Tang X, Yin G, Komaki R, Varella-Garcia M, Hong WK, Aldape KD and Wistuba II: HER family receptor abnormalities in lung cancer brain metastases and corresponding primary tumors. Clin Cancer Res 15: 4829-4837, 2009.

30. Travis WD, Brambilla E, Noguchi M, Nicholson AG, Geisinger KR, Yatabe Y, Beer DG, Powell CA, Riely GJ, Van Schil PE, et al: International association for the study of lung cancer/American thoracic society/European respiratory society international multidisciplinary classification of lung adenocarcinoma. J Thorac Oncol 6: 244-285, 2011.

31. Tsuta K, Kawago M, Inoue E, Yoshida A, Takahashi F, Sakurai H, Watanabe S, Takeuchi M, Furuta K, Asamura $\mathrm{H}$ and Tsuda $\mathrm{H}$ : The utility of the proposed IASLC/ATS/ERS lung adenocarcinoma subtypes for disease prognosis and correlation of driver gene alternations. Lung Cancer 81: 371-376, 2013.

32. Koga K, Hamasaki M, Kato F, Aoki M, Hayashi H, Iwasaki A, Kataoka $\mathrm{H}$ and Nabeshima K: Association of c-Met phosphorylation with micropapillary pattern and small cluster invasion in pT1-size lung adenocarcinoma. Lung Cancer 82: 413-419, 2013.

33. Zhou C, Wu YL, Chen G, Feng J, Liu XQ, Wang C, Zhang S, Wang J, Zhou S, Ren S, et al: Erlotinib versus chemotherapy as first-line treatment for patients with advanced EGFR mutation-positive non-small-cell lung cancer (OPTIMAL, CTONG-0802): A multicenter, open-label, randomized, phase 3 study. Lancet Oncol 12: 735-742, 2011. 
34. Welsh JW, Komaki R, Amini A, Munsell MF, Unger W, Allen PK, Chang JY, Wefel JS, McGovern SL, Garland LL, et al: Phase II trial of erlotinib plus concurrent whole-brain radiation therapy for patients with brain metastases from non-small-cell lung cancer. J Clin Oncol 31: 895-902, 2013.

35. Stanic K, Zwitter M, Hitij NT, Kern I, Sadikov A and Cufer T: Brain metastases in lung adenocarcinoma: Impact of EGFR mutation status on incidence and survival. Radiol Oncol 48 173-183, 2014

36. Lee DW, Shin DY, Kim JW, Keam B, Kim TM, Kim HJ, Kim DW, Wu HG, Paek SH, Kim YW, et al: Additional prognostic role of EGFR activating mutations in lung adenocarcinoma patients with brain metastasis: Integrating with lung specific GPA score. Lung Cancer 86: 363-368, 2014.
37. Sekine A, Satoh H, Iwasawa T, Tamura K, Hayashihara K, Saito T, Kato T, Arai M, Okudela K, Ohashi K and Ogura T: Prognostic factors for brain metastases from non-small cell lung cancer with EGFR mutation: Influence of stable extracranial disease and erlotinib therapy. Med Oncol 31: 228, 2014.

38. Tanriverdi T, Kemerdere R, Baran O, Sayyahmelli S, Ozlen F, Isler C, Uzan M and Ozyurt E: Long-term surgical and seizure outcomes of frontal low-grade gliomas. Int J Surg 33: 60-64, 2016.

(i) 9 This work is licensed under a Creative Commons EY No No Attribution-NonCommercial-NoDerivatives 4.0 International (CC BY-NC-ND 4.0) License. 